\title{
High expression levels and localization of Sox 5 in dilated cardiomyopathy
}

\author{
YAFENG LIU, BEN JIANG，YIDE CAO, WEN CHEN, LI YIN, YUEYUE XU and ZHIBING QIU \\ Department of Thoracic and Cardiovascular Surgery, Nanjing First Hospital, \\ Nanjing Medical University, Nanjing, Jiangsu 210006, P.R. China
}

Received November 18, 2019; Accepted April 15, 2020

DOI: $10.3892 / \mathrm{mmr} .2020 .11180$

\begin{abstract}
Dilated cardiomyopathy (DCM) is a disease that can lead to heart expansion and severe heart failure, but the specific pathogenesis remains unclear. Sox 5 is a member of the Sox family with a key role in cardiac function. However, the role of Sox 5 in DCM remains unclear. In the present study, wild-type mice were intraperitoneally injected with doxorubicin (Dox) to induce DCM, and heart specimens from human patients with DCM were used to investigate the preliminary role of Sox 5 in DCM. The present study demonstrated that, compared with control human hearts, the hearts of patients with DCM exhibited high expression levels of Sox 5 and activation of the wnt $/ \beta$-catenin pathway. This result was consistent with Dox-induced DCM in mice. Furthermore, in Dox-treated mice, apoptosis was activated during the development of DCM. Inflammation and collagen deposition also increased in DCM mice. The results of the present study indicate that Sox 5 may be associated with the development of DCM. Sox 5 may be a novel potential factor that regulates DCM.
\end{abstract}

\section{Introduction}

Dilated cardiomyopathy (DCM) is characterized by left ventricular dilation, which is associated with systolic and diastolic dysfunction (1). Schultheiss et al (2) reported that the prevalence of DCM in Minnesota, USA, is estimated at 1 case per 250 people. In 2010, the estimated mortality rate associated with cardiomyopathy was 5.9 deaths per 100,000 global population (2). The mechanism of DCM is complex, and there is evidence to indicate that inflammatory reactions and cardiac remodeling play a key role in compensatory repair following heart failure $(3,4)$. Furthermore, cardiomyocyte apoptosis occurs during the inflammatory process in early

Correspondence to: Professor Zhibing Qiu, Department of Thoracic and Cardiovascular Surgery, Nanjing First Hospital, Nanjing Medical University, 68 Changle Road, Nanjing, Jiangsu 210006, P.R. China

E-mail: qiuzhibing2009@163.com

Key words: Sox 5, dilated cardiomyopathy, doxorubicin, apoptosis, wnt/ $\beta$-catenin pathway heart failure and compensatory ventricular remodeling (5-9). Doxorubicin (Dox) is an anthracycline derivative that is an effective treatment for a number of soft and solid types of human malignancy. However, a number of studies have used doxorubicin to induce DCM in mice $(10,11)$.

Sox 5, a member of the Sox family of transcription factors, has a key function in the regulation of embryonic development and determination of cell fate (12). Previous studies have demonstrated that Sox 5 can promote cell proliferation in gastric and lung cancer, as well as glioma and breast tumors (13-16). In addition, Sox 5 has also been demonstrated to regulate cartilage formation (17). Previous studies have demonstrated that Sox 5 is associated with the electrocardiographic PR interval, a higher resting heart rate, atrial fibrillation and left ventricular mass (18-21). These results indicate that Sox 5 may play a key role in cardiac function (22). However, to the best of our knowledge, the role of Sox 5 in DCM has not previously been identified.

In the present study, wild-type mice were intraperitoneally injected with Dox to induce DCM, and heart specimens from human patients with DCM were used to investigate the role of Sox 5 in DCM. Furthermore, these results indicate that Sox 5 is upregulated in DCM and may be involved in the progression of DCM by modulating wnt- $1 / \beta$-catenin signaling. To our best knowledge, the present study provides the first evidence for an association between Sox 5 expression levels and DCM. This may provide a new target for interventions in patients with DCM.

\section{Materials and methods}

Human tissue. The present study was performed at the Department of Thoracic and Cardiovascular Surgery, Nanjing First Hospital, Nanjing Medical University (Nanjing, China). Left ventricular tissues of heart transplant recipients with DCM were collected, and mismatched left ventricular tissues were collected from donors as the normal control group from the Nanjing first hospital from 2013 to 2019 (normal: Male: Female, 2:1, Age, 51 2 years; DCM: Male: Female, 1:2, age, $60 \pm 5$ years). Patients with rheumatic heart disease, infectious endocarditis, inflammatory disease, underlying genetic syndromes and other causes of DCM were excluded. The left ventricular tissues were cut into two pieces (section thickness, $4 \mathrm{~mm}$ ): One was fixed in $4 \%$ formalin at room temperature for 
$12 \mathrm{~h}$ and embedded in paraffin, and the other was frozen in liquid nitrogen at $-196^{\circ} \mathrm{C}$. The protocols of all human studies were approved by the Ethics Committee of Nanjing First Hospital and were performed in accordance with the relevant guidelines and regulations. All patients provided informed written consent. The study was performed according to the Declaration of Helsinki (2000).

Animals and experimental protocols. C57BL/6 mice (male; 8 weeks old;22-27 g; $\mathrm{n}=12$ ) were obtained from the Institutional Animal Care and Use Committee of Nanjing Medical University (Nanjing, China). Animals received humane care and the mice experiments in the present study were approved by the Institutional Animal Care and Use Committee of Nanjing Medical University (approval no. SYXK2016-0006). The mice were randomly assigned to two groups $(n=6)$ : Sham or DCM group and kept in pathogen-free conditions with a $12 / 12 \mathrm{~h}$ light/dark cycle, $25^{\circ} \mathrm{C}$, with ad libitum access to food and water. In the DCM group, each mouse was injected with a cumulative dose of $25 \mathrm{mg} / \mathrm{kg}$ doxorubicin (Sigma-Aldrich; Merck KGaA) via five intraperitoneal (i.p.) injections $(5 \mathrm{mg} / \mathrm{kg}$ i.p.) over 30 days at regular intervals. The sham group received the same volume of sterile isotonic saline at the same time points. All mice were sacrificed under anesthesia, and the hearts were immediately harvested.

Echocardiographic evaluation. Mice were anesthetized using $1.5-2.0 \%$ isoflurane by inhalation, and echocardiography was performed using a Vevo2100 (VisualSonics, Inc.) ultrasound with a $30 \mathrm{MHz}$ linear array ultrasound transducer. The left ventricular internal diameter in diastole (LVIDd), left ventricular internal diameter in systole (LVIDs), left ventricular ejection fraction (EF) and fractional shortening (FS) were measured from M-mode tracings with a sweep speed of $50 \mathrm{~mm} / \mathrm{s}$ at the mid-papillary muscle level. The systole and diastole phases corresponded to the smallest and largest LV diameters, respectively. Echocardiographic measurements were taken in triplicate in M-mode from $>3$ individual mice per group.

Hematoxylin-eosin (HE) and Masson's staining. Mouse hearts were removed, immediately immersed in $4 \%$ neutral phosphate-buffered paraformaldehyde for $12 \mathrm{~h}$ at room temperature, embedded in paraffin, and sectioned ( $4 \mu \mathrm{m}$-thick). Sections were stained with HE or Masson's trichrome at the room temperature for $5 \mathrm{~min}$ and were observed to identify morphological changes and fibrosis in the myocardium under a light microscope.

Total RNA extraction and reverse transcription-quantitative $P C R(R T-q P C R)$. Total RNA was extracted from mouse heart tissues using TRIzol ${ }^{\circledR}$ (Invitrogen; Thermo Fisher Scientific, Inc.). Equal quantities of RNA $(1 \mu \mathrm{g})$ were transcribed into cDNA using the PrimeScript ${ }^{\mathrm{TM}}$ RT Reagent kit with gDNA Eraser (Takara). Quantitative TaqMan PCR assays were performed with SYBR Premix Ex TaqTM II (Takara) using the Applied Biosystems 7500 Real Time PCR System. The thermocycling conditions were as follows: $95^{\circ} \mathrm{C}$ for $15 \mathrm{~min}$, followed by 40 cycles $\left(94^{\circ} \mathrm{C}\right.$ for $15 \mathrm{sec} ; 60^{\circ} \mathrm{C}$ for $32 \mathrm{sec} ; 72^{\circ} \mathrm{C}$ for $60 \mathrm{sec}$ ). All data were normalized to the level of GAPDH and are expressed as the fold increase relative to expression levels in a sham control mouse. Primers used for quantitative real-time PCR were as follows: Atriopeptin (ANP): Forward, 5'-AAGAACCTGCTAGACCACCTGGAG-3' and reverse, 5'-TGCTTCCTCAGTCTGCTCACTCAG-3'; brain natriuretic peptide (BNP): Forward, 5'-GGAAGTCCTAGCCAGTCT CCAGAG-3' and reverse, 5'-GCCTTGGTCCTTCAAGAG CTGTC-3'; collagen 1: Forward, 5'-TGGTCCTGCTGGTCC TGCTG-3' and reverse, 5'-CTGTCACCTTGTTCGCCTGTC TC-3'; Collagen 3: Forward, 5'-TCTCCTGGTGCTGCTGGT CAC-3' and reverse, 5'-TCCATGTGGTCCAACTGGTCC TC-3'; TNF- $\alpha$ : Forward, 5'-ACGGCATGGATCTCAAAG AC-3' and reverse, 5'-AGATAGCAAATCGGCTGACG-3'; TGF- $\beta 1$ : Forward, 5'-GCA ACAATTCCTGGCGTTACC TTG-3' and reverse, 5'-CAGCCACTGCCGTACAACTCC-3'; IL-6: Forward, 5'-ACAACGATGATGCACTTGCAGA-3' and reverse, 5'-GATGAATTGGATGGTCTTGGTC-3'; and IL-10: Forward, 5'-GCTCTTACTGACTGGCATGAG-3' and reverse, 5'-CGCAGCTCTAGGAGCATGTG-3'.

Immunohistochemical (IHC) staining. Human and mouse heart tissues collected for morphological analysis were prepared as 4- $\mu$ m-thick serial paraffin-embedded sections and rehydrated in graded alcohol for $1 \mathrm{~h}$ at room temperature. The sections were treated with $3 \%$ hydrogen peroxide for $15 \mathrm{~min}$ at $37^{\circ} \mathrm{C}$ to block endogenous peroxidase activity and incubated in goat serum at room temperature for $1.5 \mathrm{~h}$ (OriGene Technologies, Inc.) to prevent non-specific binding of antibodies. The sections were then incubated separately for $14 \mathrm{~h}$ at $4^{\circ} \mathrm{C}$ with antibodies against Sox5 (anti-human,1:100; cat. no. 26041; Abcam; anti-mouse,1:100; cat. no. 13216-1-AP; Proteintech) and wnt-1 (1:100; cat. no. 15251; Abcam) and then with goat anti-rabbit IgG (cat. no. KIT-5004; MXB) for $1 \mathrm{~h}$ at $37^{\circ} \mathrm{C}$ in a humidified box. The signal of each antibody was developed using diaminobenzidine (DAB; OriGene Technologies, Inc.) substrate. The sections were counterstained with hematoxylin at room temperature for $6 \mathrm{~min}$, and images were captured using a ZEISS microscope with an A1 camera. The IHC results were evaluated based on the Fromowitz semi-quantitative analysis score, which evaluated the brown chromogen intensity (range, 0-7). The average score of each slice, as determined by two independent observers, was used for later comparisons.

TUNEL staining. Frozen mice ventricular tissues embedded in optical cutting temperature compound were cut into $4 \mu \mathrm{m}$-thick sections and fixed in $4 \%$ paraformaldehyde at room temperature for $16 \mathrm{~min}$. The TUNEL assay was performed according to the instructions of the in situ apoptosis detection kit (Roche Diagnostics (Shanghai) Co., Ltd.) and examined using a fluorescence microscope. Images were captured using Olympus BX-51 light microscope (magnification, x200). Only nuclei that were clearly located in cardiac myocytes were considered. The apoptotic index was calculated as the percentage of the number of TUNEL-positively stained nuclei to the number of DAPI- stained nuclei from 8 random fields.

Western blotting analysis. Total protein samples $(30 \mu \mathrm{g})$ were extracted from left ventricular tissues and separated by $5 \%$ SDS-PAGE. The proteins were transferred to PVDF membranes (EMD Millipore), which were washed twice 

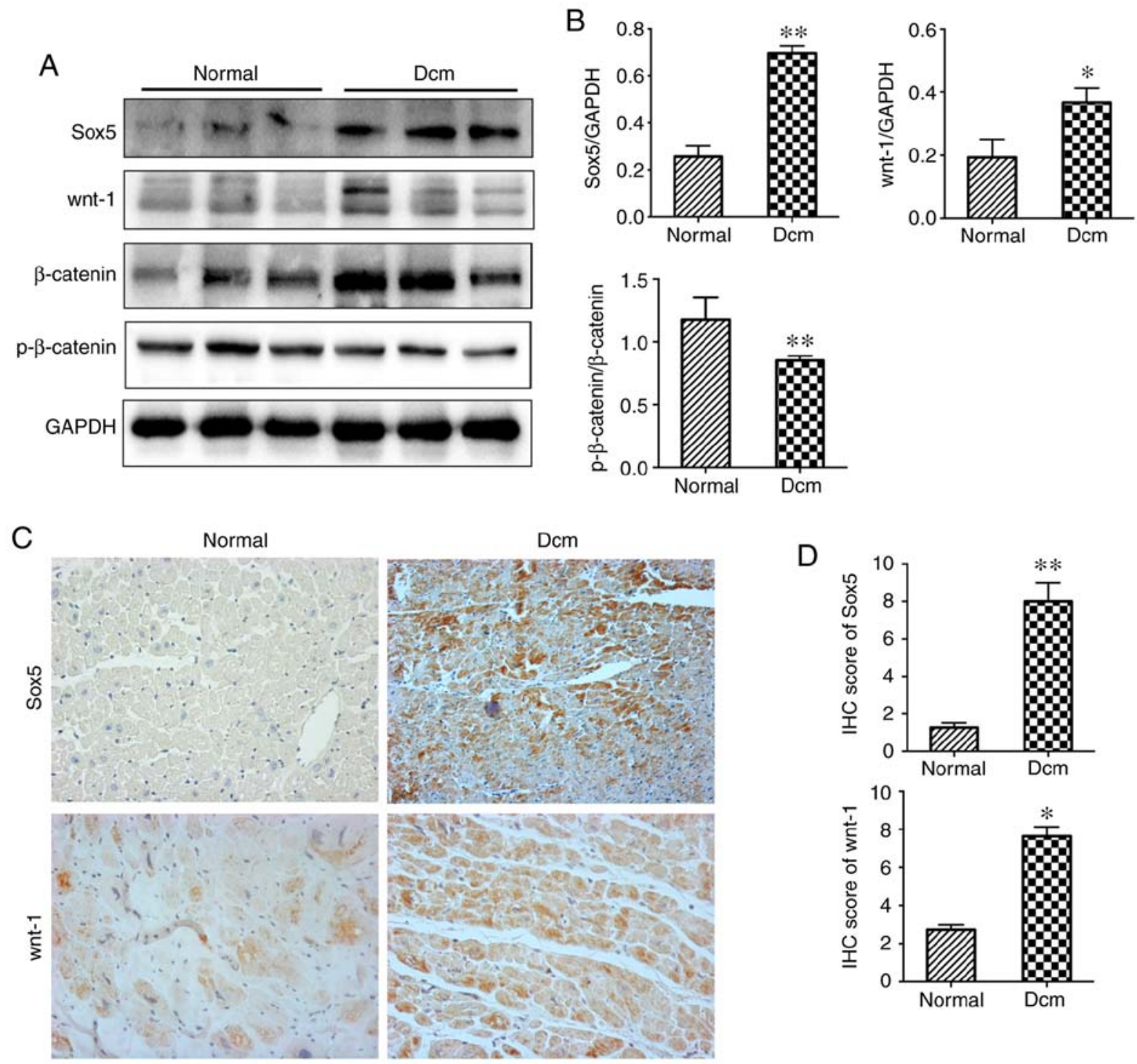

Figure 1. High expression levels of Sox 5 and activation of the wnt/ $\beta$-catenin pathway in the hearts of patients with DCM. (A) Representative images of western blots of Sox 5 and wnt/ $\beta$-catenin pathway-associated proteins (magnification, x200) in human hearts. (B) Quantitative analysis of western blots. (C) Representative images of IHC staining of Sox 5 and wnt-1 (magnification, $\mathrm{x} 400$ ). (D) Quantitative analysis of IHC staining. $\mathrm{n}=6 .{ }^{*} \mathrm{P}<0.05$; ${ }^{* *} \mathrm{P}<0.01$ vs. normal control. DCM, dilated cardiomyopathy; IHC, immunohistochemistry; WT, wild type; Dox, doxorubicin; p, phosphorylated.

in TBS with Tween-20 (1:1,000; Promega Corporation) for 10 min each and blocked with TBST containing 5\% BSA (Sigma-Aldrich; Merck $\mathrm{KGaA}$ ) for $1 \mathrm{~h}$ at $4^{\circ} \mathrm{C}$. The membranes were incubated with the following primary antibodies in TBST with Tween plus 5\% BSA overnight at $4^{\circ} \mathrm{C}$ : Anti-sox 5 (anti-human, 1:100; cat. no. 26041; Abcam; anti-mouse, 1:100; cat. no. 13216-1-AP; ProteinTech Group, Inc.)), anti-wnt-1 (1:1,000; cat. no. 15251 ; Abcam), anti- $\beta$-catenin [1:1,000; cat. no. 8480s; Cell Signaling Technology, Inc. (CST)], anti-phosphorylated $\beta$-catenin $(1: 1,000$; cat . no. 4176s; CST), anti-bax (1:1,000; cat. no. 2772s; CST), anti-cleaved-caspase3 (1:1,000; cat. no. 9661s; CST), anti-caspase 9 (1:1,000; cat. no. 9508T, CST) and horseradish peroxidase (HRP)-conjugated monoclonal mouse anti-GAPDH (1:5,000; cat. no. KC-5G5; Kangchen BioTech Co., Ltd.).. The PVDF membranes were washed with TBST (Tween-20, 1:1,000; Promega Corporation) three times for 10 min each. Subsequently, the PVDF membranes were incubated with goat anti-mouse IgG/HRP (1:5,000, cat. no. bs-0296G-HRP; BIOSS), goat anti-rabbit IgG or an HRP-conjugated antibody (1:5,000; cat. no. 7074P2; Cell Signaling Technology, Inc.) at room temperature for $1 \mathrm{~h}$. Specific proteins were detected using Immobilon Western Chemiluminescent HRP Substrate (EMD Millipore) and images were captured using a Chemi Scope system (3300 Mini; Clinx Science Instruments Co., Ltd.). The results were analyzed using Chemi Analysis Software (Clinx Science Instruments Co., Ltd.) by semi-quantifying the mean gray value of each blot. All results are representative of at least three independent experiments.

Statistical analysis. Data are presented as the mean \pm standard error of the mean. Differences among groups were assessed using analysis of variance followed by Tukey's post-hoc test. Comparisons between two groups were performed using paired Student's t test. All statistical analyses were performed using SPSS software (version 17.0; SPSS, Inc.). $\mathrm{P}<0.05$ was considered to indicate a statistically significant difference. 
A

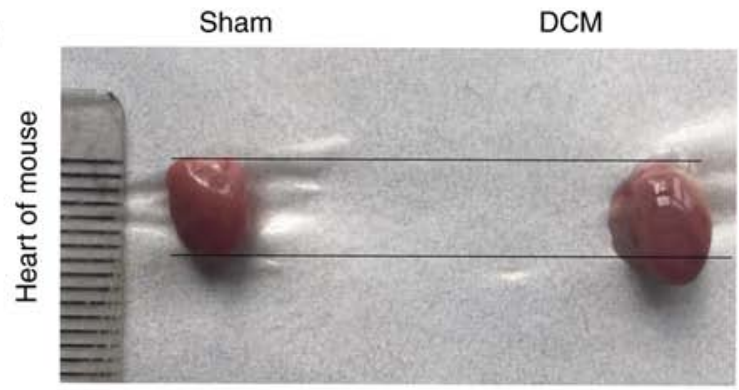

C

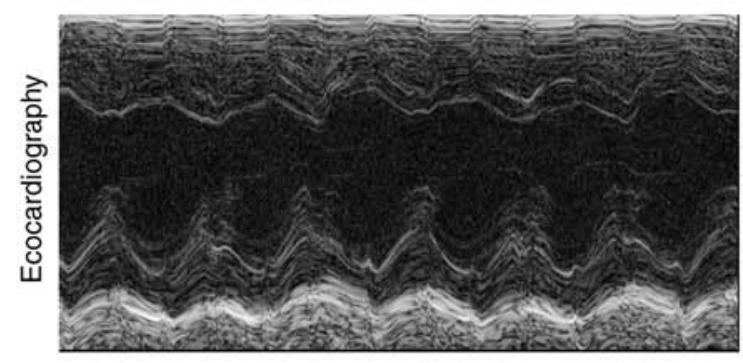

B

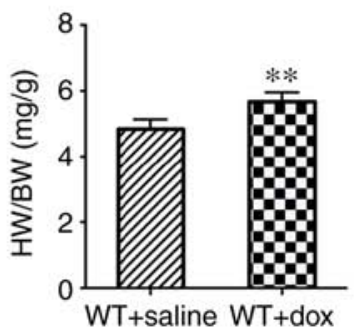

DCM
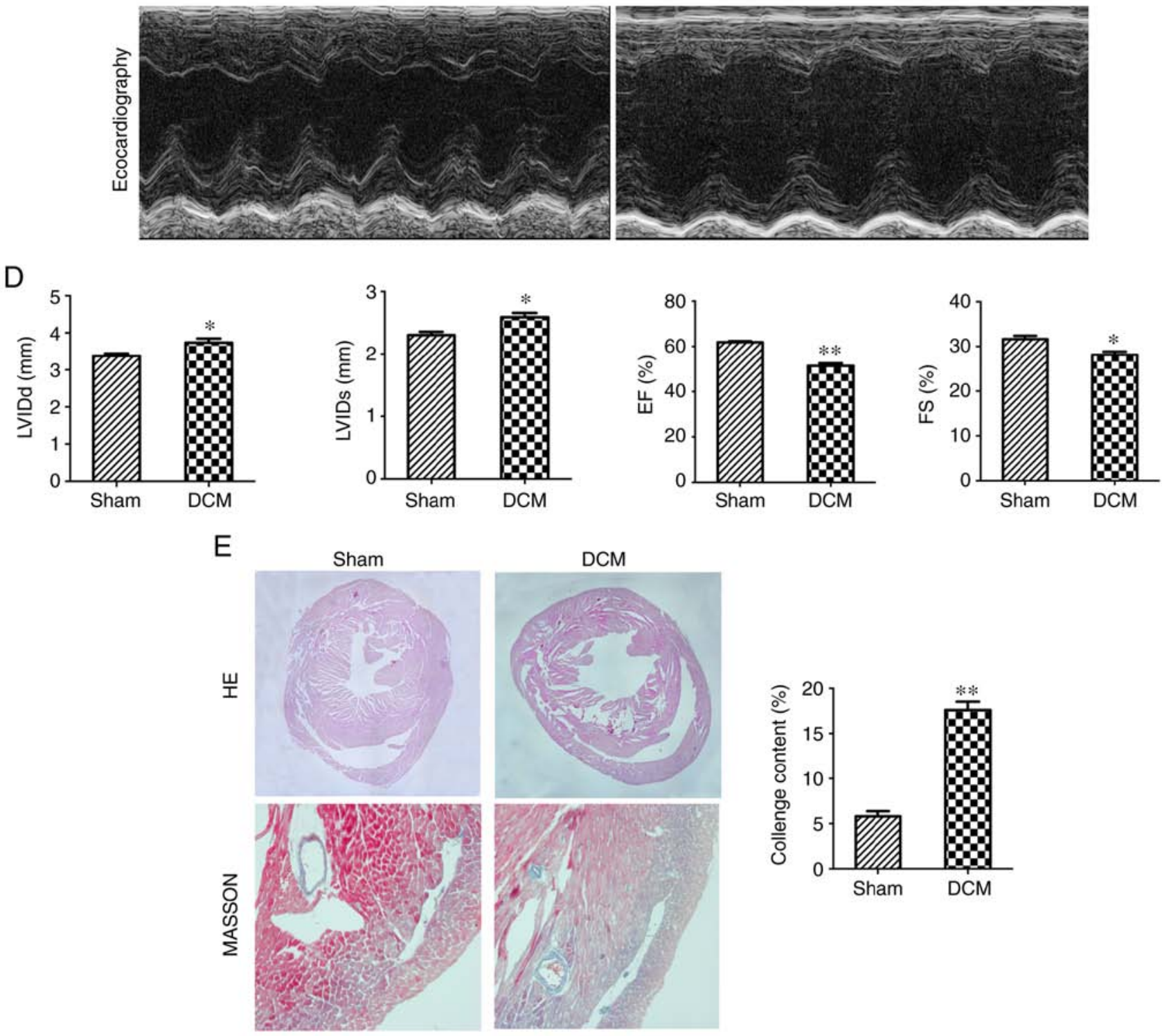

Figure 2. Dox-induced cardiac dilatation and left ventricular dysfunction in mice. (A) Representative images of the hearts of sham and DCM mice. (B) Analysis of heart/body weight. (C and D) Representative echocardiographs and echocardiographic parameters of WT mice following Dox treatment. (E) Representative images of HE and Masson's staining of tissues and analysis of collagen content. $\mathrm{n}=6$. ${ }^{*} \mathrm{P}<0.05,{ }^{* *} \mathrm{P}<0.01$ vs. sham group. Dox, doxorubicin; DCM, dilated cardiomyopathy; WT, wild type; HE, hematoxylin-eosin; LVIDd, left ventricular internal diameter in diastole; LVIDs, left ventricular internal diameter in systole; EF, ejection fraction; FS, fractional shortening.

\section{Result}

Expression levels of Sox 5 and activation of the wnt/ $\beta$-catenin pathway in the hearts of patients with DCM. Myocardial tissue samples were selected from five patients with DCM. The mismatched left ventricular tissues of donors were collected as the normal control group. Western blotting analysis demonstrated that the expression level of Sox 5 was increased in DCM heart tissue compared with the normal control heart tissue (Fig. 1A). Images of IHC staining demonstrated that the expression level (Fig. 1C) and localization of Sox 5 was the same in cardiomyocytes in samples from both groups. The present 

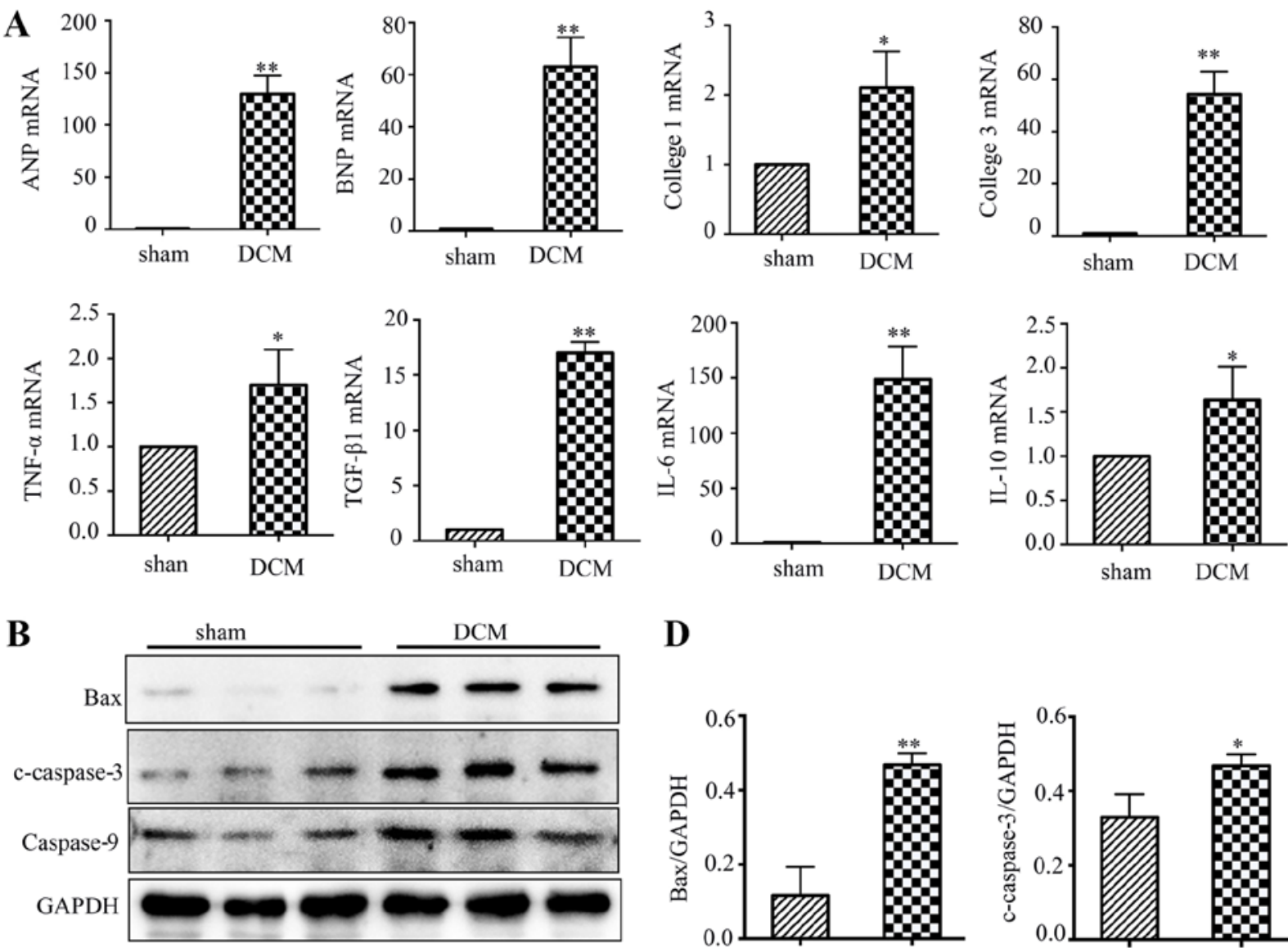

D
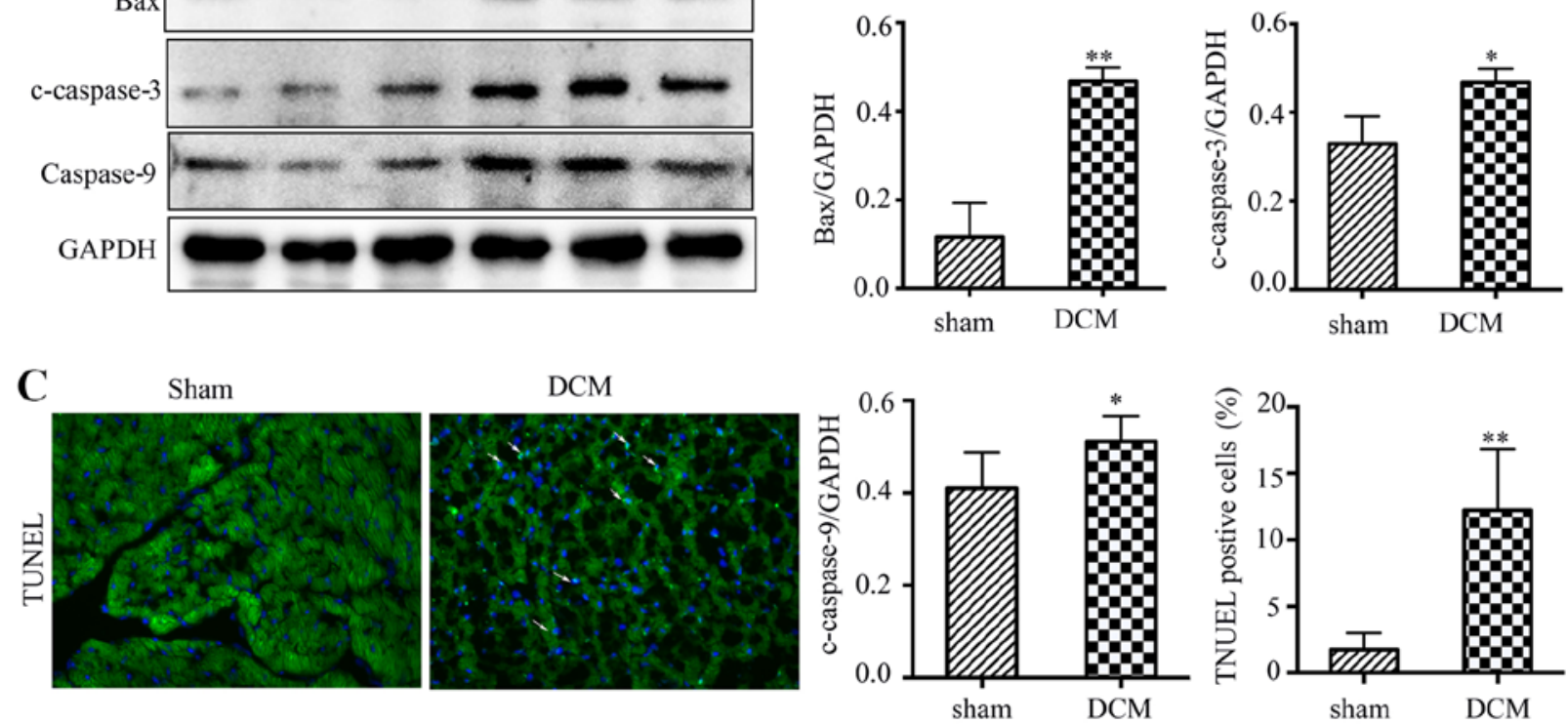

Figure 3. Inflammatory reactions, fibrosis and apoptosis are activated in the hearts of Dox-treated mice. (A) The relative mRNA levels of heart failure markers (ANP and BNP), fibrosis markers (collagen 1 and collagen 3), pro-inflammatory factors (IL-6 and TNF- $\alpha$ ) and anti-inflammatory factors (TGF- $\beta$ and IL-10). (B) Representative western blotting of Bax, c-caspase3 and caspase9 expression levels in heart tissues from WT mice following drug injection. (C) Representative images of TUNEL staining (magnification, x200) of heart tissues from WT mice following drug injection. (D) Quantitative analyses of western blots and TUNEL staining. $(\mathrm{n}=6) .{ }^{*} \mathrm{P}<0.05 ;{ }^{* *} \mathrm{P}<0.01$ vs. sham group. Dox, doxorubicin; ANP, atriopeptin; BNP, brain natriuretic peptide; WT, wild type; DCM, dilated cardiomyopathy.

study also demonstrated that the expression levels of wnt-1 and $\beta$-catenin were increased in DCM hearts, while the expression level of $\mathrm{p}$ - $\beta$-catenin was decreased, as determined by western blotting (Fig. 1A). IHC demonstrated that the expression levels of wnt- 1 were the same in both groups (Fig. 1C). The results demonstrated that Sox 5 and $w n t / \beta$-catenin pathway-associated proteins are highly expressed in DCM.

Dox-induced cardiac dilatation and left ventricular dysfunction in mice. In order to further investigate the role of Sox 5 in DCM, a mouse model of Dox-induced DCM was used. Gross anatomical analysis demonstrated that the hearts of mice treated with Dox were larger than those of mice treated with saline (Fig. 2A). Furthermore, the heart-to-body weight ratio of the sham group was lower than that of the DCM group (Fig. 2B). The EF and FS were notably decreased in the DCM group compared with the sham group; in contrast, the LVIDd and LVIDs were notably increased in the DCM group (Fig. 2C and D). HE staining of the hearts demonstrated that the sham group exhibited an increased myocardial thickness and decreased cardiac chamber diameter compared with those of the DCM group. Masson's trichrome staining demonstrated that the collagen content was notably elevated in the DCM group (Fig. 2E). These results demonstrated that mice treated with Dox exhibited pathological changes associated with DCM.

Inflammatory reactions, fibrosis and apoptosis were increased in the hearts of Dox-treated mice. DCM is associated with 
A
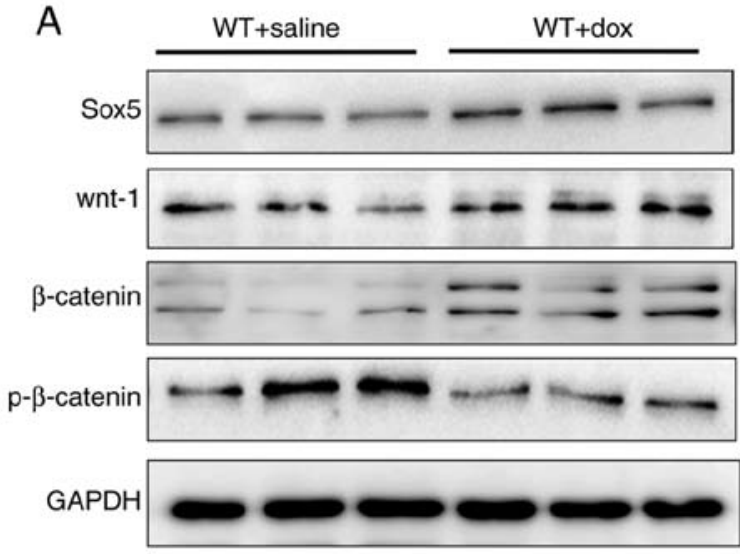

B
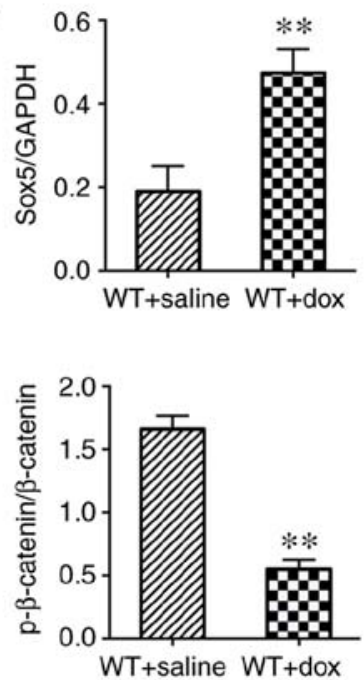
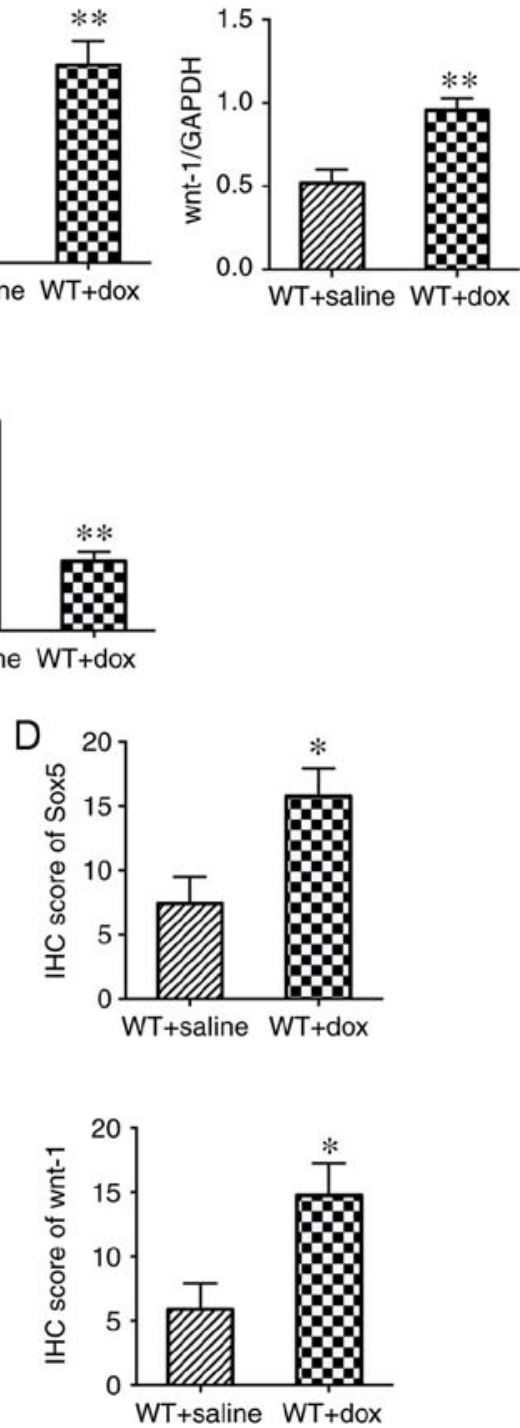

Figure 4. Increased expression levels of Sox 5 and activation of the wnt/ $\beta$-catenin pathway in hearts of Dox-induced mice. (A) Representative images of the western blotting of Sox 5 and wnt/ $/$-catenin pathway-associated proteins. (B) Quantitative analysis of western blots. (C) Representative images of IHC staining of Sox 5 and wnt-1 (magnification, $\mathrm{x} 400$ ) in heart tissues from WT mice following drug injection. (D) Quantitative analysis of IHC staining. $\mathrm{n}=6$. ${ }^{*} \mathrm{P}<0.05$; ${ }^{* *} \mathrm{P}<0.01$ vs. sham group. Dox, doxorubicin; IHC, immunohistochemistry; WT, wild type.

acute myocardial inflammation and heart failure (1). RT-qPCR analysis of heart tissues demonstrated a notable increase in heart failure markers (ANP and BNP), pro-inflammatory factors (IL-6 and TNF- $\alpha$ ) and anti-inflammatory factors (TGF- $\beta$ and IL-10) in the DCM group compared with the sham group. The present study also found that the mRNA levels of fibrosis markers (collagen 1 and 3) were increased in the DCM group (Fig. 3A), which was consistent with the results of Masson's trichrome staining. In addition, the present study tested the expression levels of apoptosis-associated proteins in mouse heart tissues. The western blot results showed that the expression levels of pro-apoptotic genes, including Bax, cleaved-caspase 3 and caspase 9, were increased in the DCM group. Furthermore, the number of TUNEL-positive nuclei was notably increased in the DCM group (Fig. 3B-D).

Increased expression levels of Sox 5 and activation of the $w n t / \beta$-catenin pathway in the hearts of Dox-treated mice. High expression levels of Sox 5 and activation of the wnt/ $\beta$-catenin pathway have been reported in the hearts of human patients with DCM (Fig. 1). Western blotting and IHC analysis demonstrated increased expression levels of Sox 5 and wnt- 1 in the DCM group compared with the sham group (Fig. 4A and C). In addition, increased expression levels of $\beta$-catenin and decreased expression levels of $p-\beta$-catenin were exhibited in the DCM group (Fig. 4A). In conclusion, Sox 5 is upregulated in DCM and may be involved in the development of DCM via modulation of Wnt-1/ $\beta$-catenin signaling.

\section{Discussion}

DCM is a type of heart failure characterized by ventricular dilatation. In the absence of hypertension, coronary artery or valvular disease, DCM is the most significant and third most common cause of heart failure and treatment requires heart transplantation (23). However, the pathogenesis of DCM is not clear. Sox 5 can downregulate the cyclin D1/cyclin-dependent kinase 4 complex to regulate cell 
proliferation and apoptosis $(24,25)$. It can also upregulate the protein expression levels of $\mathrm{N}$-cadherin, vimentin and fibronectin to promote fibrosis (17). Sox 5 is expressed in numerous human tissues, including the testis, liver, lung, fetal brain and heart (26). Liu et al (27) demonstrated that overexpression of Sox 5 can promote inflammatory responses and reverse microRNA-193a-3p mimic-mediated decrease in chondrocyte apoptosis in human osteoarthritis. However, studies have demonstrated that inhibiting Sox 5 can promote apoptosis in rheumatoid arthritis and lung cancer $(24,25)$. Axelsson et al (28) demonstrated that Sox5 protein is present both in the nucleus and in the cytosol of pancreatic sections. Stolt et al (29) demonstrated that the transcription factor Sox 5 modulates Sox 10 function during melanocyte development and that Sox 5 is expressed in the nuclei of murine melanocytes. In the present study, Sox 5 was highly expressed in DCM myocardial tissue compared with normal myocardial tissue, and histological manifestations, such as severe inflammatory responses, collagen deposition and apoptosis, were increased in DCM. Therefore, it was hypothesized that Sox 5 may be a protective factor in DCM, and its specific role will be further studied in Sox5-knockout mice. Previous studies have indicated that Sox 5 plays a key role in heart function (18-21). In the present study, western blotting analysis demonstrated a notable increase in the expression level of Sox 5 in the hearts of patients with DCM; this was confirmed via IHC. To the best of our knowledge, these results are the first to demonstrate that Sox 5 expression level is associated with DCM.

Dox is associated with dose-dependent cardiotoxicity, which can progress to heart failure. Administration of Dox at doses $>1 \mathrm{mg} / \mathrm{kg}$ results in a decreased survival rate and classic signs of DCM (30). In order to study the effect of Sox 5 on dilated cardiomyopathy in vivo, the present study constructed a mouse model of DCM via i.p. injection of Dox into wild-type mice. In the present study, the hearts of mice treated with Dox were larger, and the heart-to-body weight ratio of the DCM group was higher than that of the sham group. The body weight of mice treated with Dox was significantly decreased compared with that of mice treated with saline. In addition, following Dox treatment, the mice became thin and responded poorly. Furthermore, the mRNA levels of heart failure markers (ANP and BNP) were significantly increased, as determined by PCR, which indicated that mice treated with Dox experienced heart failure. Moreover, the Dox-treated mice exhibited pathological manifestations of DCM. HE and Masson's trichrome staining demonstrated that Dox-treated mice exhibited dilated ventricles, disordered arrangement of cardiac cells, collagen deposition and fibrosis. The echocardiography results indicated that Dox-treated mice exhibited an increased ventricular inner diameter, a decreased ejection fraction and heart failure consistent with DCM.

Inflammation is involved in the pathogenesis of DCM. DCM is associated with inflammation, as documented by increased levels of inflammatory cytokines, such as IL-6, IL-10 and TNF- $\alpha$ (31). PCR analysis of the expression levels of pro-inflammatory factors in the DCM group were consistent with this. Collagen deposition and ventricular remodeling, which maintain the function of the heart, are important during the progression of DCM $(32,33)$. Consistent with the Masson's trichrome staining results, RT-qPCR demonstrated that the expression levels of collagen (collagen 1 and collagen 3 ) were increased in the DCM group compared with the sham group. Apoptosis is a form of programmed cell death controlled by numerous genes that maintains the stability of the internal environment (34). Previous studies have demonstrated that cell apoptosis promotes the progression of Dox-induced DCM and may be the molecular mechanism underlying DCM (35). The cysteine aspartate protein (caspase) family participates in the regulation of cell apoptosis. Caspase-9, which is an initiator of apoptosis, triggers an executioner caspase (caspase 3 or 7), ensuring continuity of the process (34). In the present study, TUNEL staining and analysis of Bax, cleaved-caspase 3 and caspase 9 expression levels using western blotting demonstrated a notable increase in apoptosis in the DCM group compared with the sham group.

Wnt proteins are a family of secreted cysteine-rich glycoproteins involved in a number of cellular processes, including proliferation, differentiation, senescence and apoptosis. The $w n t / \beta$-catenin pathway has been demonstrated to play a role in DCM (36). In previous studies, Sox 5 has been demonstrated to regulate the wnt/ $\beta$-catenin pathway $(37,38)$. $\beta$-catenin is the key effector of the wnt/ $\beta$-catenin pathway and functions as a transcriptional co-activator that is critical for target gene expression level. In the absence of wnt ligands, $\beta$-catenin is bound by the scaffold protein Axin, which facilitates its phosphorylation by glycogen synthase kinase 3-b via a destruction complex. When wnt ligands bind to the Frizzled and low-density lipoprotein receptor $5 / 6$ complex, the $\beta$-catenin destruction complex becomes dysfunctional $(37,38)$. However, the mechanism of this process is not fully understood. When the wnt $/ \beta$-catenin pathway is activated, the expression level of $\beta$-catenin in cytosol increases, and $\beta$-catenin translocates to the nucleus to form a complex with the transcription factor TCF/LEF, leading to the activation of target genes $(39,40)$. The present study demonstrated that the expression levels of wnt $/ \beta$-catenin pathway-associated proteins were altered in DCM. In light of the expression level pattern of Sox 5 and the reported association between the wnt/ $/ 3$-catenin pathway and Sox 5 , it was hypothesized that sox 5 may be associated with the wnt/ $\beta$-catenin pathway during the development of DCM.

In conclusion, the present study demonstrated that Sox 5 may be associated with acute inflammation, apoptosis, collagen deposition and ventricular remodeling during the development of DCM.

\section{Acknowledgements}

Not applicable.

\section{Funding}

The present study was supported by grants from the Natural Science Foundation of Nanjing Provincial Level in 2018 (grant no. BK20181119), the Postgraduate Research \& Practice Innovation Program of Jiangsu Province (grant no. SJCX19_0328) and the Jiangsu Provincial Special Program of Medical Science (no. BE2017610).

\section{Availability of data and materials}

All data generated or analyzed during this study are included in this published article. 


\section{Authors' contributions}

YL designed the experiment and wrote the manuscript. BJ constructed the mouse model. YC, LY and YX performed mechanism research and molecular biological detection. WC and ZQ conceptualized the study. All authors read and approved the final manuscript.

\section{Ethics approval and consent to participate}

The present study was approved by the Ethics Committee of Nanjing First Hospital (approval no. KY20190404-03-KS-01) and were performed in accordance with the relevant guidelines and regulations.

\section{Patient consent for publication}

All patients provided informed written consent.

\section{Competing interests}

The authors declare that they have no competing interests.

\section{References}

1. Jefferies JL and Towbin JA: Dilated cardiomyopathy. Lancet 375: 752-762, 2010.

2. Schultheiss HP, Fairweather D, Caforio ALP, Escher F, Hershberger RE, Lipshultz SE, Liu PP, Matsumori A, Mazzanti A, McMurray J and Priori SG: Dilated cardiomyopathy. Nat Rev Dis Primers 5: 32, 2019.

3. Cutler MJ, Jeyaraj D and Rosenbaum DS: Cardiac electrical remodeling in health and disease. Trends Pharmacol Sci 32: 174-180, 2011

4. Mann DL: Inflammatory mediators and the failing heart: Past, present, and the foreseeable future. Circ Res 91: 988-998, 2002.

5. Crow MT, Mani K, Nam YJ and Kitsis RN: The mitochondrial death pathway and cardiac myocyte apoptosis. Circ Res 95 957-970, 2004.

6. Hikoso S,Ikeda Y, Yamaguchi O, Takeda T, Higuchi Y,Hirotani S, Kashiwase K, Yamada M, Asahi M, Matsumura Y, et al: Progression of heart failure was suppressed by inhibition of apoptosis signal-regulating kinase 1 via transcoronary gene transfer. J Am Coll Cardiol 50: 453-462, 2007.

7. Huang Q, Zhou HJ,Zhang H, Huang Y, Hinojosa-Kirschenbaum F, Fan P, Yao L, Belardinelli L, Tellides G, Giordano FJ, et al: Thioredoxin-2 inhibits mitochondrial reactive oxygen species generation and apoptosis stress kinase-1 activity to maintain cardiac function. Circulation 131: 1082-1097, 2015.

8. Maejima Y, Kyoi S, Zhai P, Liu T, Li H, Ivessa A, Sciarretta S, Del Re DP, Zablocki DK, Hsu CP, et al: Mst1 inhibits autophagy by promoting the interaction between Beclin1 and Bcl-2. Nat Med 19: 1478-1488, 2013.

9. Miller EJ, Li J, Leng L, McDonald C, Atsumi T, Bucala R and Young LH: Macrophage migration inhibitory factor stimulates AMP-activated protein kinase in the ischaemic heart. Nature 451: 578-582, 2008

10. Hong YJ, Kim TK, Hong D, Park CH, Yoo SJ, Wickum ME, Hur J, Lee HJ, Kim YJ, Suh YJ, et al: Myocardial characterization using dual-energy CT in doxorubicin-induced DCM: Comparison with CMR T1-mapping and histology in a rabbit model. JACC Cardiovasc Imaging 9: 836-845, 2016.

11. Marechal X, Montaigne D, Marciniak C, Marchetti P, Hassoun SM, Beauvillain JC, Lancel S and Neviere R: Doxorubicin-induced cardiac dysfunction is attenuated by ciclosporin treatment in mice through improvements in mitochondrial bioenergetics. Clin Sci (Lond) 121: 405-413, 2011.

12. Lefebvre V: The SoxD transcription factors-Sox 5, Sox6, and Sox13-are key cell fate modulators. Int J Biochem Cell Biol 42: 429-432, 2010
13. Zou H, Wang S, Wang S, Wu H, Yu J, Chen Q, Cui W, Yuan Y, Wen X, He J, et al: SOX5 interacts with YAP1 to drive malignant potential of non-small cell lung cancer cells. Am J Cancer Res 8: 866-878, 2018.

14. Sun C, Ban Y, Wang K, Sun Y and Zhao Z: SOX5 promotes breast cancer proliferation and invasion by transactivation of EZH2. Oncol Lett 17: 2754-2762, 2019.

15. Ueda R, Yoshida K, Kawase T, Kawakami Y and Toda M: Preferential expression and frequent IgG responses of a tumor antigen, SOX5, in glioma patients. Int J Cancer 120: 1704-1711, 2007.

16. You J, Zhao Q, Fan X and Wang J: SOX5 promotes cell invasion and metastasis via activation of Twist-mediated epithelial-mesenchymal transition in gastric cancer. Onco Targets Ther 12: 2465-2476, 2019.

17. Hattori T, Coustry F, Stephens S, Eberspaecher H, Takigawa M, Yasuda $\mathrm{H}$ and de Crombrugghe B: Transcriptional regulation of chondrogenesis by coactivator Tip60 via chromatin association with Sox9 and Sox5. Nucleic Acids Res 36: 3011-3024, 2008.

18. Pfeufer A, van Noord C, Marciante KD, Arking DE, Larson MG, Smith AV, Tarasov KV, Müller M, Sotoodehnia N, Sinner MF, et al: Genome-wide association study of PR interval. Nat Genet 42: 153-159, 2010.

19. Eijgelsheim M, Newton-Cheh C, Sotoodehnia N, de Bakker PI, Müller M, Morrison AC, Smith AV, Isaacs A, Sanna S, Dörr M, et al: Genome-wide association analysis identifies multiple loci related to resting heart rate. Hum Mol Genet 19: 3885-3894, 2010.

20. Olesen MS, Holst AG, Jabbari J, Nielsen JB, Christophersen IE, Sajadieh A, Hauns $\varnothing$ S and Svendsen JH: Genetic loci on chromosomes $4 \mathrm{q} 25,7 \mathrm{p} 31$, and $12 \mathrm{p} 12$ are associated with onset of lone atrial fibrillation before the age of 40 years. Can J Cardiol 28: 191-195, 2012.

21. Della-Morte D, Beecham A, Rundek T, Wang L, McClendon MS, Slifer S, Blanton SH, Di Tullio MR and Sacco RL: A follow-up study for left ventricular mass on chromosome 12p11 identifies potential candidate genes. BMC Med Genet 12: 100, 2011.

22. Li A, Ahsen OO, Liu JJ, Du C, McKee ML, Yang Y, Wasco W, Newton-Cheh CH, O'Donnell CJ, Fujimoto JG, et al: Silencing of the Drosophila ortholog of SOX5 in heart leads to cardiac dysfunction as detected by optical coherence tomography. Hum Mol Genet 22: 3798-3806, 2013.

23. Halliday BP, Cleland JGF, Goldberger JJ and Prasad SK: Personalizing risk stratification for sudden death in dilated cardiomyopathy: The past, present, and future. Circulation 136: 215-231, 2017.

24. Liu Y, Zhang XL, Li XF, Tang YC and Zhao X: miR-212-3p reduced proliferation, and promoted apoptosis of fibroblast-like synoviocytes via down-regulating SOX5 in rheumatoid arthritis. Eur Rev Med Pharmacol Sci 22: 461-471, 2018.

25. Li G, Wang K, Wang J, Qin S, Sun X and Ren H: miR-497-5p inhibits tumor cell growth and invasion by targeting SOX5 in non-small-cell lung cancer. J Cell Biochem 120: 10587-10595, 2019.

26. Veronique MW, Critcher R, Ashworth A and Goodfellow PN: Cloning and characterization of SOX5, a new member of the human SOX gene family. Genomics 36: 354-358, 1996.

27. Liu F, Liu X, Yang Y, Sun Z, Deng S, Jiang Z, Li W and Wu F: NEAT1/miR-193a-3p/SOX5 axis regulates cartilage matrix degradation in human osteoarthritis. Cell Biol Int 44: 947-957, 2020.

28. Axelsson AS, Mahdi T, Nenonen HA, Singh T, Hänzelmann S, Wendt A, Bagge A, Reinbothe TM, Millstein J, Yang X, et al: Sox 5 regulates beta-cell phenotype and is reduced in type 2 diabetes. Nat Commun 8: 15652, 2017.

29. Stolt CC, Lommes P, Hillgartner S and Wegner M: The transcription factor Sox 5 modulates Sox 10 function during melanocyte development. Nucleic Acids Res 36: 5427-5440, 2008.

30. Hayward R and David SH: Doxorubicin Cardiotoxicity in the rat: An in vivo characterization. J Am Assoc Lab Anim Sci 46: 20-32,2007.

31. Sun X, Shan A, Wei Z and Xu B: Intravenous mesenchymal stem cell-derived exosomes ameliorate myocardial inflammation in the dilated cardiomyopathy. Biochem Biophys Res Commun 503: 2611-2618, 2018

32. Iwata Y, Ohtake H, Suzuki O, Matsuda J, Komamura K and Wakabayashi S: Blockade of sarcolemmal TRPV2 accumulation inhibits progression of dilated cardiomyopathy. Cardiovasc Res 99: 760-768, 2013. 
33. Japp AG, Gulati A, Cook SA, Cowie MR and Prasad SK: The diagnosis and evaluation of dilated cardiomyopathy. J Am Coll Cardiol 67: 2996-3010, 2016.

34. Grilo AL and Mantalaris A: Apoptosis: A mammalian cell bioprocessing perspective. Biotechnol Adv 37: 459-475, 2019.

35. Kankeu C, Clarke K, Passante E and Huber HJ: Doxorubicin-induced chronic dilated cardiomyopathy-the apoptosis hypothesis revisited. J Mol Med (Berl) 95: 239-248, 2016.

36. Le Dour C, Macquart C, Sera F, Homma S, Bonne G, Morrow JP, Worman HJ and Muchir A: Decreased WNT/ $\beta$-catenin signalling contributes to the pathogenesis of dilated cardiomyopathy caused by mutations in the lamin a/C gene. Hum Mol Genet 26 : 333-343, 2017.

37. Martinez-Morales PL, Quiroga AC, Barbas JA and Morales AV: SOX 5 controls cell cycle progression in neural progenitors by interfering with the WNT-beta-catenin pathway. EMBO Rep 11: 466-472, 2010.
38. Li A, Hooli B, Mullin K, Tate RE, Bubnys A, Kirchner R, Chapman B, Hofmann O, Hide W and Tanzi RE: Silencing of the Drosophila ortholog of SOX5 leads to abnormal neuronal development and behavioral impairment. Hum Mol Genet 26: 1472-1482, 2017.

39. Nusse $\mathrm{R}$ and Clevers $\mathrm{H}$ : Wnt/ $\beta$-catenin signaling, disease, and emerging therapeutic modalities. Cell 169: 985-999, 2017.

40. Moon RT, Kohn AD, De Ferrari GV and Kaykas A: WNT and beta-catenin signalling: diseases and therapies. Nat Rev Genet 5: 691-701, 2004.

This work is licensed under a Creative Commons Attribution-NonCommercial-NoDerivatives 4.0 International (CC BY-NC-ND 4.0) License. 